\title{
ON THE INCLINATION OF THE LUNAR AXIS
}

\author{
M. MOUTSOULAS
}

Department of Astronomy, University of Manchester, Manchester, England

Since the days of Cassini (1693), study of the position of the lunar axis relative to an inertial system of reference has been based on the assumption that "the inclination of the Moon's equator to the plane of the ecliptic is constant". And, although the actual value of that inclination has been subject to continuous changes and modifications, reduced from the originally suggested $4 \frac{1}{2}^{\circ}$ to $1^{\circ} 32^{\prime} 4^{\prime \prime}$ (Koziel, 1967), no sufficient attention has been paid to the fact that, as the lunar globe moves within the field of varying external forces, the inclination of its axis cannot remain constant.

Moreover, certain confusion seems to have been involved in works dealing with the subject, and several 'axes' of the Moon appear to be interchanged with each other and used quite inconsistently. It must be understood that the shortest inertial axis of the lunar dynamical configuration, the instantaneous axis of rotation of the Moon, and the rotation axis which the Moon would possess if it could obey precisely Cassini's laws of motion, do not coincide with each other (Habibullin, 1968). Therefore, whenever measurements of reference points of the lunar surface are reduced for the determination of the position of the lunar 'axis', either the selenographic coordinates of those points or the inclination of the axis they are referred to, should be expressed as time-dependent functions. Which one would be considered as constant depends on the definition; but they cannot both possess constant values.

The Eulerian equations of motion provide the required relations between the system of principal axes of the Moon, Oxyz, and a system fixed in space. If we adopt as our fixed system the ecliptic system of coordinates and we represent the longitude of the descending node of the Moon's equator, the angular distance of the direction $O x$ from the descending node of the Moon's equator, and the inclination of the lunar equator to the ecliptic, with $\psi, \varphi$ and $\theta$, respectively, the kinematic equations take the form:

$$
\begin{aligned}
& \omega_{x}=-\frac{\mathrm{d} \psi}{\mathrm{d} t} \sin \theta \sin \varphi-\frac{\mathrm{d} \theta}{\mathrm{d} t} \cos \varphi \\
& \omega_{y}=-\frac{\mathrm{d} \psi}{\mathrm{d} t} \sin \theta \cos \varphi+\frac{\mathrm{d} \theta}{\mathrm{d} t} \sin \varphi \\
& \omega_{z}=\frac{\mathrm{d} \psi}{\mathrm{d} t} \cos \theta+\frac{\mathrm{d} \varphi}{\mathrm{d} t} .
\end{aligned}
$$

where $\omega_{x}, \omega_{y}$ and $\omega_{z}$ stand for the components of the angular velocity along the $x$, $y, z$ axes. 
The system of Equations (1)-(3) solved for $\mathrm{d} \psi / \mathrm{d} t, \mathrm{~d} \varphi / \mathrm{d} t$ and $\mathrm{d} \theta / \mathrm{d} t$ gives:

$$
\begin{aligned}
& \frac{\mathrm{d} \psi}{\mathrm{d} t}=-\frac{\omega_{x} \sin \varphi+\omega_{y} \cos \varphi}{\sin \theta} \\
& \frac{\mathrm{d} \varphi}{\mathrm{d} t}=\frac{\left(\omega_{x} \sin \varphi+\omega_{y} \cos \varphi\right) \cos \theta}{\sin \theta}+\omega_{z} \\
& \frac{\mathrm{d} \theta}{\mathrm{d} t}=\omega_{y} \sin \varphi-\omega_{x} \cos \varphi .
\end{aligned}
$$

We can introduce into the right-hand side of the Equations (4)-(6) the components $\sigma, \tau$ and $\varrho$ of the physical libration, which, as is known, represent deviations in the position of the $O x y z$ system from that prescribed by Cassini's laws, and therefore are connected to the angles $\psi, \varphi$ and $\theta$ by means of the relations:

$$
\begin{aligned}
& \psi=\Omega+\sigma \\
& \varphi=180^{\circ}+l-\psi+\tau \\
& \theta=I+\varrho .
\end{aligned}
$$

where $\Omega$ is the longitude of the ascending node of the lunar orbit, $l$ the mean longitude of the Moon and $I$ the mean value of the inclination of the lunar equator to the ecliptic.

Retaining the first-order terms for the small quantities $\sigma, \tau$ and $\varrho$, and taking into account the relation:

$$
l=g+\omega+\Omega
$$

which expresses the mean longitude of the Moon in terms of the mean anomaly of the Moon, $g$, the angular distance of the Moon's perigee from the ascending node of the orbit, $\omega$, and the longitude of the ascending node of the lunar orbit, $\Omega$, we find the system of equations:

$$
\begin{aligned}
& \frac{\mathrm{d} \psi}{\mathrm{d} t}=\frac{1}{\sin I+\varrho \cos I} \times \\
& \quad \times\left\{\left[\omega_{x} \cos (g+\omega)-\omega_{y} \sin (g+\omega)\right](\tau-\sigma)+\omega_{x} \sin (g+\omega)+\omega_{y} \cos (g+\omega)\right\}
\end{aligned}
$$

$$
\begin{aligned}
\frac{\mathrm{d} \varphi}{\mathrm{d} t} & =-\frac{\cos I}{\sin I+\varrho \cos I} \times \\
& \times\left\{\left[\omega_{x} \cos (g+\omega)-\omega_{y} \sin (g+\omega)\right](\tau-\sigma)+\omega_{x} \sin (g+\omega)+\omega_{y} \cos (g+\omega)\right\} \\
& +\frac{\sin I}{\sin I+\varrho \cos I}\left[\omega_{x} \sin (g+\omega)+\omega_{y} \cos (g+\omega)\right] \varrho+\omega_{z}
\end{aligned}
$$

$$
\frac{\mathrm{d} \theta}{\mathrm{d} t}=\left[\omega_{x} \sin (g+\omega)+\omega_{y} \cos (g+\omega)\right](\sigma-\tau)+\omega_{x} \cos (g+\omega)-\omega_{y} \sin (g+\omega)
$$


or the equivalent to that system:

$$
\begin{aligned}
\frac{\mathrm{d} \sigma}{\mathrm{d} t}=\frac{1}{\sin I} & +\varrho \cos I \\
& \times\left\{\left[\omega_{x} \cos (g+\omega)-\omega_{y} \sin (g+\omega)\right] \times\right. \\
& \left.\times(\tau-\sigma)+\omega_{x} \sin (g+\omega)+\omega_{y} \cos (g+\omega)\right\}-\frac{\mathrm{d} \Omega}{\mathrm{d} t}
\end{aligned}
$$

$$
\begin{aligned}
& \frac{\mathrm{d} \tau}{\mathrm{d} t}=\frac{1-\cos I}{\sin I+\varrho \cos I}\left\{\left[\omega_{x} \cos (g+\omega)-\omega_{y} \sin (g+\omega)\right] \times\right. \\
& \left.\times(\tau-\sigma)+\omega_{x} \sin (g+\omega)+\omega_{y} \cos (g+\omega)\right\}+\frac{\sin I}{\sin I+\varrho \cos I} \times \\
& \times\left[\omega_{x} \sin (g+\omega)+\omega_{y} \cos (g+\omega)\right] \varrho+\omega_{z}-\frac{\mathrm{d} g}{\mathrm{~d} t}-\frac{\mathrm{d} \omega}{\mathrm{d} t}-\frac{\mathrm{d} \Omega}{\mathrm{d} t}
\end{aligned}
$$

$\frac{\mathrm{d} \varrho}{\mathrm{d} t}=\left[\omega_{x} \sin (g+\omega)+\omega_{y} \cos (g+\omega)\right](\sigma-\tau)+\omega_{x} \cos (g+\omega)-\omega_{y} \sin (g+\omega)$.

Solution of the system of Equations (11)-(13), or its equivalent (14)-(16), will give the value of the true inclination, $\theta$, of the Moon's axis to that of the ecliptic, as a function of time.

The velocity components $\omega_{x}, \omega_{y}$ and $\tilde{\omega}_{z}$ are derived from Euler's dynamical equations:

$$
\begin{aligned}
& A \frac{\mathrm{d} \omega_{x}}{\mathrm{~d} t}=(B-C)\left(\omega_{y} \omega_{z}-\frac{3 G m_{\oplus}}{R^{5}} y_{E} z_{E}\right) \\
& B \frac{\mathrm{d} \omega_{y}}{\mathrm{~d} t}=(C-A)\left(\omega_{z} \omega_{x}-\frac{3 G m_{\oplus}}{R^{5}} z_{E} x_{E}\right) \\
& C \frac{\mathrm{d} \omega_{z}}{\mathrm{~d} t}=(A-B)\left(\omega_{x} \omega_{y}-\frac{3 G m_{\oplus}}{R^{5}} x_{E} y_{E}\right)
\end{aligned}
$$

where $A, B, C$ are the principal moments of inertia of the Moon, $G$ is the gravitational constant, $m_{\oplus}$ is the mass of the Earth, $R$ the distance between the centre of mass of the Moon and that of the Earth, and $x_{E}, y_{E}, z_{E}$ the rectangular coordinates of the centre of the Earth in the selenocentric system $O x y z$, which are related to the true selenocentric longitude of the Earth, $v$, the true geocentric latitude of the Moon, $B_{\mathbb{1}}$, and the Eulerian angles, by means of the expressions:

$$
\begin{aligned}
& x_{E}=R \cos B_{\mathbb{Q}}\left[\cos (v-\varphi)-\sin v \sin \varphi(1-\cos \theta)+\tan B_{\mathbb{Q}} \sin \varphi \sin \theta\right], \\
& y_{E}=R \cos B_{\mathbb{Q}}\left[\sin (v-\varphi)-\sin v \cos \varphi(1-\cos \theta)+\tan B_{\mathbb{Q}} \cos \varphi \sin \theta\right], \\
& z_{E}=R \cos B_{\mathbb{Q}}\left[\sin v \sin \theta-\tan B_{\mathbb{Q}} \cos \theta\right] .
\end{aligned}
$$

As is known, the true geocentric latitude of the Moon, $B_{\mathfrak{l}}$, can be expressed in terms 
of the inclination of the lunar orbit to the ecliptic, $i$, and the true selenocentric longitude of the Earth, $v$, by means of the formula:

$$
\tan B_{\varangle}=-\tan i \sin v .
$$

We can, moreover, express the coordinates $x_{E}, y_{E}, z_{E}$ in terms of the true geocentric longitude and latitude of the Moon, $L$ and $B_{\mathbb{f}}$, its mean anomaly, $g$, the angular distance of the lunar perigee from the ascending node of the orbit, $\omega$, and the physical libration components $\sigma, \tau, \varrho$, taking into account the relations (7)-(9) and the fact that

$$
v=180^{\circ}+L+\psi \text {. }
$$

Then the system of Equations (17)-(19) takes the form:

$$
\begin{aligned}
\frac{\mathrm{d} \omega_{x}}{\mathrm{~d} t}+\alpha \omega_{y} \omega_{z} & =\frac{3 G m_{\oplus} \alpha \cos ^{2} B_{(}}{R^{3}}\{\sigma[\sin (L-l) \cdot \cos (L-l+g+\omega)- \\
& -(1-\cos I+\tan i \sin I)\left\{\frac{1}{2} \sin 2(L-l+g+\omega) \cos (g+\omega)+\right. \\
& +\sin (L-l+g+\omega) \cos (L-l+2 g+2 \omega)\}] \sin (I+i)+ \\
& +\tau[\cos (L-l) \sin (L-l+g+\omega)-(1-\cos I+\tan i \sin I) \times \\
& \left.\times \sin ^{2}(L-l+g+\omega) \sin (g+\omega)\right] \sin (I+i)+\varrho\{[(1-\cos I+ \\
& +\tan i \sin I) \sin ^{2}(L-l+g+\omega) \cos (g+\omega)-\sin (L-l) \times \\
& \times \sin (L-l+g+\omega)] \cos (I+i)+(\sin I+\tan i \cos I) \sin (I+i) \times \\
& \left.\times \sin { }^{2}(L-l+g+\omega) \cos (g+\omega)\right\}+[(1-\cos I+\tan i \sin I) \times \\
& \times \sin { }^{2}(L-l+g+\omega) \cos (g+\omega)-\sin (L-l) \times \\
& \times \sin (L-l+g+\omega)] \sin (I+i)\} \\
\frac{\mathrm{d} \omega_{y}}{\mathrm{~d} t}-\beta \omega_{z} \omega_{x} & =-\frac{3 G m_{\oplus} \beta \cos ^{2} B_{(}\{\sigma[\cos (L-l) \cos (L-l+g+\omega)-}{R^{3}} \\
& -(1-\cos I+\tan i \sin I)\left\{\frac{1}{2} \sin ^{2}(L-l+g+\omega) \sin (g+\omega)+\right. \\
& +\sin (L-l+2 g+2 \omega) \sin (L-l+g+\omega)\}] \sin (I+i)- \\
& -\tau[\sin (L-l) \sin (L-l+g+\omega)-(1-\cos I+\tan i \sin I) \times \\
& \left.\times \sin { }^{2}(L-l+g+\omega) \cos (g+\omega)\right] \sin (I+i)+ \\
& +\varrho\left[\left\{(1-\cos I+\tan i \sin I) \sin ^{2}(L-l+g+\omega) \sin (g+\omega)-\right.\right. \\
& -\cos (L-l) \sin (L-l+g+\omega)\} \cos (I+i)+(\sin I+\tan i \cos I) \times \\
& \left.\times \sin (I+i) \sin { }^{2}(L-l+g+\omega) \sin (g+\omega)\right]+ \\
& +\left[(1-\cos I+\tan i \sin I) \sin ^{2}(L-l+g+\omega) \sin (g+\omega)-\right. \\
& -\cos (L-l) \sin (L-l+g+\omega)] \sin (I+i)\} \\
& +3 G m_{\oplus}\left(L \cos ^{2} B\right.
\end{aligned}
$$

$$
\frac{\mathrm{d} \omega_{z}}{\mathrm{~d} t}+\gamma \omega_{x} \omega_{y}=\frac{3 G m_{\oplus} \gamma \cos ^{2} B_{\llbracket}}{2 R^{3}}\{2 \sigma(1-\cos I+\tan i \sin I) \cos 2(g+\omega)-
$$$$
-2 \tau[(\cos I-\tan i \sin I) \cos 2(L-l)-(1-\cos I+\tan i \sin I) \times
$$$$
\times \cos 2(g+\omega)]-\varrho(\sin I+\tan i \cos I)[\sin 2(L-l)+\sin 2(g+\omega)]+
$$$$
+(\cos I-\tan i \sin I) \sin 2(L-l)-
$$$$
-(1-\cos I+\tan i \sin I) \sin 2(g+\omega)\}
$$ 
where $\alpha, \beta$ and $\gamma$ represent the ratios of mechanical ellipticities of the Moon:

$$
\alpha=(C-B) / A, \quad \beta=(C-A) / B, \quad \gamma=(B-A) / C .
$$

We are therefore led to the simultaneous solution of the Equations (14), (15), (16), (25), (26) and (27), which will produce the values of the corrections $\varrho$ for the inclination of the Moon's shortest axis of inertia to the ecliptic, as well as the libration components $\sigma$ and $\tau$.

The variation in the inclination of the lunar axis during the year 1971, obtained by numerical integration of this system of equations, is presented in Table I. It has been assumed that at the beginning of the year the librations were zero and the inclination possessed the mean value of $1^{\circ} 32^{\prime} 4^{\prime \prime}$. In order to establish an ephemeris for the inclination, it now only remains to obtain by observational detection a set of measurements which will provide the correct initial conditions for the solution, while the periodic nature and other general characteristics of the nutational motion of the lunar axis are apparent in the values presented here.

TABLE I

Variation of the inclination of the lunar axis to the ecliptic over the period of a year

\begin{tabular}{|c|c|c|c|}
\hline Day & Inclination & Day & Inclination \\
\hline 1 & $1^{\circ} 32^{\prime} 4^{\prime \prime}$ & 28 & $1^{\circ} 32^{\prime} 5^{\prime \prime}$ \\
\hline 2 & $1^{\circ} 32^{\prime} 1^{\prime \prime}$ & 29 & $1^{\circ} 31^{\prime} 59^{\prime \prime}$ \\
\hline 3 & $1^{\circ} 31^{\prime} 57^{\prime \prime}$ & 30 & $1^{\circ} 31^{\prime} 55^{\prime \prime}$ \\
\hline 4 & $1^{\circ} 31^{\prime} 51^{\prime \prime}$ & & \\
\hline 5 & $1^{\circ} 31^{\prime} 42^{\prime \prime}$ & 31 & $1^{\circ} 31^{\prime} 46^{\prime \prime}$ \\
\hline 6 & $1^{\circ} 31^{\prime} 31^{\prime \prime}$ & 32 & $1^{\circ} 31^{\prime} 38^{\prime \prime}$ \\
\hline 7 & $1^{\circ} 31^{\prime} 18^{\prime \prime}$ & 33 & $1^{\circ} 31^{\prime} 25^{\prime \prime}$ \\
\hline 8 & $1^{\circ} 31^{\prime} 2^{\prime \prime}$ & 34 & $1^{\circ} 31^{\prime} 13^{\prime \prime}$ \\
\hline 9 & $1^{\circ} 30^{\prime} 43^{\prime \prime}$ & 35 & $1^{\circ} 30^{\prime} 55^{\prime \prime}$ \\
\hline \multirow[t]{2}{*}{10} & $1^{\circ} 30^{\prime} 31^{\prime \prime}$ & 36 & $1^{\circ} 30^{\prime} 41^{\prime \prime}$ \\
\hline & & 37 & $1^{\circ} 30^{\prime} 23^{\prime \prime}$ \\
\hline 11 & $1^{\circ} 30^{\prime} 18^{\prime \prime}$ & 38 & $1^{\circ} 30^{\prime} 12^{\prime \prime}$ \\
\hline 12 & $1^{\circ} 30^{\prime} 10^{\prime \prime}$ & 39 & $1^{\circ} 30^{\prime} 4^{\prime \prime}$ \\
\hline 13 & $1^{\circ} 30^{\prime} 6^{\prime \prime}$ & 40 & $1^{\circ} 30^{\prime} \quad 2^{\prime \prime}$ \\
\hline 14 & $1^{\circ} 30^{\prime} 7^{\prime \prime}$ & & \\
\hline 15 & $1^{\circ} 30^{\prime} 10^{\prime \prime}$ & 41 & $1^{\circ} 30^{\prime} 5^{\prime \prime}$ \\
\hline 16 & $1^{\circ} 30^{\prime} 21^{\prime \prime}$ & 42 & $1^{\circ} 30^{\prime} 14^{\prime \prime}$ \\
\hline 17 & $1^{\circ} 30^{\prime} 34^{\prime \prime}$ & 43 & $1^{\circ} 30^{\prime} 26^{\prime \prime}$ \\
\hline 18 & $1^{\circ} 30^{\prime} 52^{\prime \prime}$ & 44 & $1^{\circ} 30^{\prime} 42^{\prime \prime}$ \\
\hline 19 & $1^{\circ} 31^{\prime} 10^{\prime \prime}$ & 45 & $1^{\circ} 31^{\prime} 0^{\prime \prime}$ \\
\hline \multirow[t]{2}{*}{20} & $1^{\circ} 31^{\prime} 30^{\prime \prime}$ & 46 & $1^{\circ} 31^{\prime} 19^{\prime \prime}$ \\
\hline & & 47 & $1^{\circ} 31^{\prime} 38^{\prime \prime}$ \\
\hline 21 & $1^{\circ} 31^{\prime} 44^{\prime \prime}$ & 48 & $1^{\circ} 31^{\prime} 55^{\prime \prime}$ \\
\hline 22 & $1^{\circ} 31^{\prime} 58^{\prime \prime}$ & 49 & $1^{\circ} 32^{\prime} 7^{\prime \prime}$ \\
\hline 23 & $1^{\circ} 32^{\prime} 4^{\prime \prime}$ & 50 & $1^{\circ} 32^{\prime} 16^{\prime \prime}$ \\
\hline 24 & $1^{\circ} 32^{\prime} 11^{\prime \prime}$ & & \\
\hline 25 & $1^{\circ} 32^{\prime} 11^{\prime \prime}$ & 51 & $1^{\circ} 32^{\prime} 20^{\prime \prime}$ \\
\hline 26 & $1^{\circ} 32^{\prime} 12^{\prime \prime}$ & 52 & $1^{\circ} 32^{\prime} 20^{\prime \prime}$ \\
\hline 27 & $1^{\circ} 32^{\prime} 8^{\prime \prime}$ & 53 & $1^{\circ} 32^{\prime} 17^{\prime \prime}$ \\
\hline
\end{tabular}


Table I (continued)

\begin{tabular}{|c|c|c|c|}
\hline Day & Inclination & Day & Inclination \\
\hline 54 & $1^{\circ} 32^{\prime} 13^{\prime \prime}$ & 102 & $1^{\circ} 32^{\prime} 12^{\prime \prime}$ \\
\hline 55 & $1^{\circ} 32^{\prime} 6^{\prime \prime}$ & 103 & $1^{\circ} 32^{\prime} 25^{\prime \prime}$ \\
\hline 56 & $1^{\circ} 32^{\prime} 0^{\prime \prime}$ & 104 & $1^{\circ} 32^{\prime} 39^{\prime \prime}$ \\
\hline 57 & $1^{\circ} 31^{\prime} 52^{\prime \prime}$ & 105 & $1^{\circ} 32^{\prime} 41^{\prime \prime}$ \\
\hline 58 & $1^{\circ} 31^{\prime} 43^{\prime \prime}$ & 106 & $1^{\circ} 32^{\prime} 44^{\prime \prime}$ \\
\hline 59 & $1^{\circ} 31^{\prime} 33^{\prime \prime}$ & 107 & $1^{\circ} 32^{\prime} 35^{\prime \prime}$ \\
\hline \multirow[t]{2}{*}{60} & $1^{\circ} 31^{\prime} 21^{\prime \prime}$ & 108 & $1^{\circ} 32^{\prime} 31^{\prime \prime}$ \\
\hline & & 109 & $1^{\circ} 32^{\prime} 16^{\prime \prime}$ \\
\hline 61 & $1^{\circ} 31^{\prime} 7^{\prime \prime}$ & 110 & $1^{\circ} 32^{\prime} 8^{\prime \prime}$ \\
\hline 62 & $1^{\circ} 30^{\prime} 53^{\prime \prime}$ & & \\
\hline 63 & $1^{\circ} 30^{\prime} 38^{\prime \prime}$ & 111 & $1^{\circ} 31^{\prime} 52^{\prime \prime}$ \\
\hline 64 & $1^{\circ} 30^{\prime} 22^{\prime \prime}$ & 112 & $1^{\circ} 31^{\prime} 43^{\prime \prime}$ \\
\hline 65 & $1^{\circ} 30^{\prime} 9^{\prime \prime}$ & 113 & $1^{\circ} 31^{\prime} 26^{\prime \prime}$ \\
\hline 66 & $1^{\circ} 30^{\prime} 0^{\prime \prime}$ & 114 & $1^{\circ} 31^{\prime} 15^{\prime \prime}$ \\
\hline 67 & $1^{\circ} 29^{\prime} 57^{\prime \prime}$ & 115 & $1^{\circ} 30^{\prime} 56^{\prime \prime}$ \\
\hline 68 & $1^{\circ} 30^{\prime} 2^{\prime \prime}$ & 116 & $1^{\circ} 30^{\prime} 43^{\prime \prime}$ \\
\hline 69 & $1^{\circ} 30^{\prime} 12^{\prime \prime}$ & 117 & $1^{\circ} 30^{\prime} 25^{\prime \prime}$ \\
\hline \multirow[t]{2}{*}{70} & $1^{\circ} 30^{\prime} 28^{\prime \prime}$ & 118 & $1^{\circ} 30^{\prime} 14^{\prime \prime}$ \\
\hline & & 119 & $1^{\circ} 30^{\prime} 1^{\prime \prime}$ \\
\hline 71 & $1^{\circ} 30^{\prime} 46^{\prime \prime}$ & 120 & $1^{\circ} 29^{\prime} 57^{\prime \prime}$ \\
\hline 72 & $1^{\circ} 31^{\prime} 8^{\prime \prime}$ & & \\
\hline 73 & $1^{\circ} 31^{\prime} 27^{\prime \prime}$ & 121 & $1^{\circ} 29^{\prime} 53^{\prime \prime}$ \\
\hline 74 & $1^{\circ} 31^{\prime} 49^{\prime \prime}$ & 122 & $1^{\circ} 29^{\prime} 57^{\prime \prime}$ \\
\hline 75 & $1^{\circ} 32^{\prime} 5^{\prime \prime}$ & 123 & $1^{\circ} 30^{\prime} 4^{\prime \prime}$ \\
\hline 76 & $1^{\circ} 32^{\prime} 21^{\prime \prime}$ & 124 & $1^{\circ} 30^{\prime} 20^{\prime \prime}$ \\
\hline 77 & $1^{\circ} 32^{\prime} 29^{\prime \prime}$ & 125 & $1^{\circ} 30^{\prime} 38^{\prime \prime}$ \\
\hline 78 & $1^{\circ} 32^{\prime} 33^{\prime \prime}$ & 126 & $1^{\circ} 31^{\prime} 3^{\prime \prime}$ \\
\hline 79 & $1^{\circ} 32^{\prime} 31^{\prime \prime}$ & 127 & $1^{\circ} 31^{\prime} 27^{\prime \prime}$ \\
\hline \multirow[t]{2}{*}{80} & $1^{\circ} 32^{\prime} 28^{\prime \prime}$ & 128 & $1^{\circ} 31^{\prime} 52^{\prime \prime}$ \\
\hline & & 129 & $1^{\circ} 32^{\prime} 11^{\prime \prime}$ \\
\hline 81 & $1^{\circ} 32^{\prime} 20^{\prime \prime}$ & 130 & $1^{\circ} 32^{\prime} 28^{\prime \prime}$ \\
\hline 82 & $1^{\circ} 32^{\prime} 12^{\prime \prime}$ & & \\
\hline 83 & $1^{\circ} 32^{\prime} 2^{\prime \prime}$ & 131 & $1^{\circ} 32^{\prime} 38^{\prime \prime}$ \\
\hline 84 & $1^{\circ} 31^{\prime} 53^{\prime \prime}$ & 132 & $1^{\circ} 32^{\prime} 47^{\prime \prime}$ \\
\hline 85 & $1^{\circ} 31^{\prime} 41^{\prime \prime}$ & 133 & $1^{\circ} 32^{\prime} 46^{\prime \prime}$ \\
\hline 86 & $1^{\circ} 31^{\prime} 30^{\prime \prime}$ & 134 & $1^{\circ} 32^{\prime} 44^{\prime \prime}$ \\
\hline 87 & $1^{\circ} 31^{\prime} 17^{\prime \prime}$ & 135 & $1^{\circ} 32^{\prime} 34^{\prime \prime}$ \\
\hline 88 & $1^{\circ} 31^{\prime} 4^{\prime \prime}$ & 136 & $1^{\circ} 32^{\prime} 24^{\prime \prime}$ \\
\hline 89 & $1^{\circ} 30^{\prime} 48^{\prime \prime}$ & 137 & $1^{\circ} 32^{\prime} 9^{\prime \prime}$ \\
\hline \multirow[t]{2}{*}{90} & $1^{\circ} 30^{\prime} 34^{\prime \prime}$ & 138 & $1^{\circ} 31^{\prime} 57^{\prime \prime}$ \\
\hline & & 139 & $1^{\circ} 31^{\prime} 41^{\prime \prime}$ \\
\hline 91 & $1^{\circ} 30^{\prime} 20^{\prime \prime}$ & 140 & $1^{\circ} 31^{\prime} 28^{\prime \prime}$ \\
\hline 92 & $1^{\circ} 30^{\prime} 10^{\prime \prime}$ & & \\
\hline 93 & $1^{\circ} 30^{\prime} 0^{\prime \prime}$ & 141 & $1^{\circ} 31^{\prime} 10^{\prime \prime}$ \\
\hline 94 & $1^{\circ} 29^{\prime} 58^{\prime \prime}$ & 142 & $1^{\circ} 30^{\prime} 55^{\prime \prime}$ \\
\hline 95 & $1^{\circ} 29^{\prime} 58^{\prime \prime}$ & 143 & $1^{\circ} 30^{\prime} 36^{\prime \prime}$ \\
\hline 96 & $1^{\circ} 30^{\prime} 8^{\prime \prime}$ & 144 & $1^{\circ} 30^{\prime} 20^{\prime \prime}$ \\
\hline 97 & $1^{\circ} 30^{\prime} 21^{\prime \prime}$ & 145 & $1^{\circ} 30^{\prime} 2^{\prime \prime}$ \\
\hline 98 & $1^{\circ} 30^{\prime} 45^{\prime \prime}$ & 146 & $1^{\circ} 29^{\prime} 53^{\prime \prime}$ \\
\hline 99 & $1^{\circ} 31^{\prime} 5^{\prime \prime}$ & 147 & $1^{\circ} 29^{\prime} 44^{\prime \prime}$ \\
\hline \multirow[t]{2}{*}{100} & $1^{\circ} 31^{\prime} 31^{\prime \prime}$ & 148 & $1^{\circ} 29^{\prime} 45^{\prime \prime}$ \\
\hline & & 149 & $1^{\circ} 29^{\prime} 48^{\prime \prime}$ \\
\hline 101 & $1^{\circ} 31^{\prime} 50^{\prime \prime}$ & 150 & $1^{\circ} 30^{\prime} 1^{\prime \prime}$ \\
\hline
\end{tabular}


Table I (continued)

\begin{tabular}{|c|c|c|c|}
\hline Day & Inclination & Day & Inclination \\
\hline 151 & $1^{\circ} 30^{\prime} 12^{\prime \prime}$ & 200 & $1^{\circ} 29^{\prime} 50^{\prime \prime}$ \\
\hline 152 & $1^{\circ} 30^{\prime} 36^{\prime \prime}$ & & \\
\hline 153 & $1^{\circ} 30^{\prime} 56^{\prime \prime}$ & 201 & $1^{\circ} 29^{\prime} 38^{\prime \prime}$ \\
\hline 154 & $1^{\circ} 31^{\prime} 26^{\prime \prime}$ & 202 & $1^{\circ} 29^{\prime} 33^{\prime \prime}$ \\
\hline 155 & $1^{\circ} 31^{\prime} 47^{\prime \prime}$ & 203 & $1^{\circ} 29^{\prime} 33^{\prime \prime}$ \\
\hline 156 & $1^{\circ} 32^{\prime} 13^{\prime \prime}$ & 204 & $1^{\circ} 29^{\prime} 43^{\prime \prime}$ \\
\hline 157 & $1^{\circ} 32^{\prime} 27^{\prime \prime}$ & 205 & $1^{\circ} 30^{\prime} 2^{\prime \prime}$ \\
\hline 158 & $1^{\circ} 32^{\prime} 43^{\prime \prime}$ & 206 & $1^{\circ} 30^{\prime} 25^{\prime \prime}$ \\
\hline 159 & $1^{\circ} 32^{\prime} 45^{\prime \prime}$ & 207 & $1^{\circ} 30^{\prime} 53^{\prime \prime}$ \\
\hline \multirow[t]{2}{*}{160} & $1^{\circ} 32^{\prime} 50^{\prime \prime}$ & 208 & $1^{\circ} 31^{\prime} 21^{\prime \prime}$ \\
\hline & & 209 & $1^{\circ} 31^{\prime} 49^{\prime \prime}$ \\
\hline 161 & $1^{\circ} 32^{\prime} 44^{\prime \prime}$ & 210 & $1^{\circ} 32^{\prime} 15^{\prime \prime}$ \\
\hline 162 & $1^{\circ} 32^{\prime} 40^{\prime \prime}$ & & \\
\hline 163 & $1^{\circ} 32^{\prime} 26^{\prime \prime}$ & 211 & $1^{\circ} 32^{\prime} 37^{\prime \prime}$ \\
\hline 164 & $1^{\circ} 32^{\prime} 16^{\prime \prime}$ & 212 & $1^{\circ} 32^{\prime} 54^{\prime \prime}$ \\
\hline 165 & $1^{\circ} 31^{\prime} 58^{\prime \prime}$ & 213 & $1^{\circ} 33^{\prime} \quad 5^{\prime \prime}$ \\
\hline 166 & $1^{\circ} 31^{\prime} 46^{\prime \prime}$ & 214 & $1^{\circ} 33^{\prime} 6^{\prime \prime}$ \\
\hline 167 & $1^{\circ} 31^{\prime} 27^{\prime \prime}$ & 215 & $1^{\circ} 33^{\prime} 3^{\prime \prime}$ \\
\hline 168 & $1^{\circ} 31^{\prime} 14^{\prime \prime}$ & 216 & $1^{\circ} 32^{\prime} 52^{\prime \prime}$ \\
\hline 169 & $1^{\circ} 30^{\prime} 53^{\prime \prime}$ & 217 & $1^{\circ} 32^{\prime} 40^{\prime \prime}$ \\
\hline \multirow[t]{2}{*}{170} & $1^{\circ} 30^{\prime} 38^{\prime \prime}$ & 218 & $1^{\circ} 32^{\prime} 23^{\prime \prime}$ \\
\hline & & 219 & $1^{\circ} 32^{\prime} 8^{\prime \prime}$ \\
\hline 171 & $1^{\circ} 30^{\prime} 17^{\prime \prime}$ & 220 & $1^{\circ} 31^{\prime} 49^{\prime \prime}$ \\
\hline 172 & $1^{\circ} 30^{\prime} 3^{\prime \prime}$ & & \\
\hline 173 & $1^{\circ} 29^{\prime} 45^{\prime \prime}$ & 221 & $1^{\circ} 31^{\prime} 32^{\prime \prime}$ \\
\hline 174 & $1^{\circ} 29^{\prime} 38^{\prime \prime}$ & 222 & $1^{\circ} 31^{\prime} 11^{\prime \prime}$ \\
\hline 175 & $1^{\circ} 29^{\prime} 35^{\prime \prime}$ & 223 & $1^{\circ} 30^{\prime} 53^{\prime \prime}$ \\
\hline 176 & $1^{\circ} 29^{\prime} 42^{\prime \prime}$ & 224 & $1^{\circ} 30^{\prime} 33^{\prime \prime}$ \\
\hline 177 & $1^{\circ} 29^{\prime} 52^{\prime \prime}$ & 225 & $1^{\circ} 30^{\prime} 17^{\prime \prime}$ \\
\hline 178 & $1^{\circ} 30^{\prime} 11^{\prime \prime}$ & 226 & $1^{\circ} 30^{\prime} 0^{\prime \prime}$ \\
\hline 179 & $1^{\circ} 30^{\prime} 32^{\prime \prime}$ & 227 & $1^{\circ} 29^{\prime} 48^{\prime \prime}$ \\
\hline \multirow[t]{2}{*}{180} & $1^{\circ} 30^{\prime} 57^{\prime \prime}$ & 228 & $1^{\circ} 29^{\prime} 38^{\prime \prime}$ \\
\hline & & 229 & $1^{\circ} 29^{\prime} 32^{\prime \prime}$ \\
\hline 181 & $1^{\circ} 31^{\prime} 23^{\prime \prime}$ & 230 & $1^{\circ} 29^{\prime} 33^{\prime \prime}$ \\
\hline 182 & $1^{\circ} 31^{\prime} 50^{\prime \prime}$ & & \\
\hline 183 & $1^{\circ} 32^{\prime} 14^{\prime \prime}$ & 231 & $1^{\circ} 29^{\prime} 41^{\prime \prime}$ \\
\hline 184 & $1^{\circ} 32^{\prime} 35^{\prime \prime}$ & 232 & $1^{\circ} 29^{\prime} 56^{\prime \prime}$ \\
\hline 185 & $1^{\circ} 32^{\prime} 47^{\prime \prime}$ & 233 & $1^{\circ} 30^{\prime} 18^{\prime \prime}$ \\
\hline 186 & $1^{\circ} 32^{\prime} 56^{\prime \prime}$ & 234 & $1^{\circ} 30^{\prime} 48^{\prime \prime}$ \\
\hline 187 & $1^{\circ} 32^{\prime} 56^{\prime \prime}$ & 235 & $1^{\circ} 31^{\prime} 17^{\prime \prime}$ \\
\hline 188 & $1^{\circ} 32^{\prime} 52^{\prime \prime}$ & 236 & $1^{\circ} 31^{\prime} 48^{\prime \prime}$ \\
\hline 189 & $1^{\circ} 32^{\prime} 43^{\prime \prime}$ & 237 & $1^{\circ} 32^{\prime} 13^{\prime \prime}$ \\
\hline \multirow{2}{*}{190} & $1^{\circ} 32^{\prime} 33^{\prime \prime}$ & 238 & $1^{\circ} 32^{\prime} 38^{\prime \prime}$ \\
\hline & & 239 & $1^{\circ} 32^{\prime} 56^{\prime \prime}$ \\
\hline 191 & $1^{\circ} 32^{\prime} 18^{\prime \prime}$ & 240 & $1^{\circ} 33^{\prime} 9^{\prime \prime}$ \\
\hline 192 & $1^{\circ} 32^{\prime} 4^{\prime \prime}$ & & \\
\hline 193 & $1^{\circ} 31^{\prime} 46^{\prime \prime}$ & 241 & $1^{\circ} 33^{\prime} 14^{\prime \prime}$ \\
\hline 194 & $1^{\circ} 31^{\prime} 30^{\prime \prime}$ & 242 & $1^{\circ} 33^{\prime} 13^{\prime \prime}$ \\
\hline 195 & $1^{\circ} 31^{\prime} 12^{\prime \prime}$ & 243 & $1^{\circ} 33^{\prime} 2^{\prime \prime}$ \\
\hline 196 & $1^{\circ} 30^{\prime} 56^{\prime \prime}$ & 244 & $1^{\circ} 32^{\prime} 49^{\prime \prime}$ \\
\hline 197 & $1^{\circ} 30^{\prime} 37^{\prime \prime}$ & 245 & $1^{\circ} 32^{\prime} 30^{\prime \prime}$ \\
\hline 198 & $1^{\circ} 30^{\prime} 21^{\prime \prime}$ & 246 & $1^{\circ} 32^{\prime} 13^{\prime \prime}$ \\
\hline 199 & $1^{\circ} 30^{\prime} 4^{\prime \prime}$ & 247 & $1^{\circ} 31^{\prime} 51^{\prime \prime}$ \\
\hline
\end{tabular}


Table I (continued)

\begin{tabular}{|c|c|c|c|}
\hline Day & Inclination & Day & Inclination \\
\hline 248 & $1^{\circ} 31^{\prime} 33^{\prime \prime}$ & 296 & $1^{\circ} 33^{\prime} 19^{\prime \prime}$ \\
\hline 249 & $1^{\circ} 31^{\prime} 10^{\prime \prime}$ & 297 & $1^{\circ} 33^{\prime} 12^{\prime \prime}$ \\
\hline \multirow[t]{2}{*}{250} & $1^{\circ} 30^{\prime} 51^{\prime \prime}$ & 298 & $1^{\circ} 33^{\prime} 3^{\prime \prime}$ \\
\hline & & 299 & $1^{\circ} 32^{\prime} 45^{\prime \prime}$ \\
\hline 251 & $1^{\circ} 30^{\prime} 28^{\prime \prime}$ & 300 & $1^{\circ} 32^{\prime} 27^{\prime \prime}$ \\
\hline 252 & $1^{\circ} 30^{\prime} 9^{\prime \prime}$ & & \\
\hline 253 & $1^{\circ} 29^{\prime} 51^{\prime \prime}$ & 301 & $1^{\circ} 32^{\prime} 3^{\prime \prime}$ \\
\hline 254 & $1^{\circ} 29^{\prime} 38^{\prime \prime}$ & 302 & $1^{\circ} 31^{\prime} 42^{\prime \prime}$ \\
\hline 255 & $1^{\circ} 29^{\prime} 28^{\prime \prime}$ & 303 & $1^{\circ} 31^{\prime} 17^{\prime \prime}$ \\
\hline 256 & $1^{\circ} 29^{\prime} 26^{\prime \prime}$ & 304 & $1^{\circ} 30^{\prime} 56^{\prime \prime}$ \\
\hline 257 & $1^{\circ} 29^{\prime} 29^{\prime \prime}$ & 305 & $1^{\circ} 30^{\prime} 32^{\prime \prime}$ \\
\hline 258 & $1^{\circ} 29^{\prime} 36^{\prime \prime}$ & 306 & $1^{\circ} 30^{\prime} 11^{\prime \prime}$ \\
\hline 259 & $1^{\circ} 29^{\prime} 52^{\prime \prime}$ & 307 & $1^{\circ} 29^{\prime} 46^{\prime \prime}$ \\
\hline \multirow[t]{2}{*}{260} & $1^{\circ} 30^{\prime} 13^{\prime \prime}$ & 308 & $1^{\circ} 29^{\prime} 29^{\prime \prime}$ \\
\hline & & 309 & $1^{\circ} 29^{\prime} 10^{\prime \prime}$ \\
\hline 261 & $1^{\circ} 30^{\prime} 41^{\prime \prime}$ & 310 & $1^{\circ} 29^{\prime} 4^{\prime \prime}$ \\
\hline 262 & $1^{\circ} 31^{\prime} 11^{\prime \prime}$ & & \\
\hline 263 & $1^{\circ} 31^{\prime} 44^{\prime \prime}$ & 311 & $1^{\circ} 29^{\prime} 4^{\prime \prime}$ \\
\hline 264 & $1^{\circ} 32^{\prime} 11^{\prime \prime}$ & 312 & $1^{\circ} 29^{\prime} 16^{\prime \prime}$ \\
\hline 265 & $1^{\circ} 32^{\prime} 37^{\prime \prime}$ & 313 & $1^{\circ} 29^{\prime} 32^{\prime \prime}$ \\
\hline 266 & $1^{\circ} 32^{\prime} 54^{\prime \prime}$ & 314 & $1^{\circ} 29^{\prime} 59^{\prime \prime}$ \\
\hline 267 & $1^{\circ} 33^{\prime} 8^{\prime \prime}$ & 315 & $1^{\circ} 30^{\prime} 27^{\prime \prime}$ \\
\hline 268 & $1^{\circ} 33^{\prime} 15^{\prime \prime}$ & 316 & $1^{\circ} 31^{\prime} 0^{\prime \prime}$ \\
\hline 269 & $1^{\circ} 33^{\prime} 16^{\prime \prime}$ & 317 & $1^{\circ} 31^{\prime} 34^{\prime \prime}$ \\
\hline \multirow[t]{2}{*}{270} & $1^{\circ} 33^{\prime} 9^{\prime \prime}$ & 318 & $1^{\circ} 32^{\prime} 7^{\prime \prime}$ \\
\hline & & 319 & $1^{\circ} 32^{\prime} 38^{\prime \prime}$ \\
\hline 271 & $1^{\circ} 32^{\prime} 57^{\prime \prime}$ & 320 & $1^{\circ} 33^{\prime} 2^{\prime \prime}$ \\
\hline 272 & $1^{\circ} 32^{\prime} 40^{\prime \prime}$ & & \\
\hline 273 & $1^{\circ} 32^{\prime} 19^{\prime \prime}$ & 321 & $1^{\circ} 33^{\prime} 18^{\prime \prime}$ \\
\hline 274 & $1^{\circ} 31^{\prime} 58^{\prime \prime}$ & 322 & $1^{\circ} 33^{\prime} 27^{\prime \prime}$ \\
\hline 275 & $1^{\circ} 31^{\prime} 36^{\prime \prime}$ & 323 & $1^{\circ} 33^{\prime} 28^{\prime \prime}$ \\
\hline 276 & $1^{\circ} 31^{\prime} 14^{\prime \prime}$ & 324 & $1^{\circ} 33^{\prime} 22^{\prime \prime}$ \\
\hline 277 & $1^{\circ} 30^{\prime} 51^{\prime \prime}$ & 325 & $1^{\circ} 33^{\prime} 9^{\prime \prime}$ \\
\hline 278 & $1^{\circ} 30^{\prime} 29^{\prime \prime}$ & 326 & $1^{\circ} 32^{\prime} 56^{\prime \prime}$ \\
\hline 279 & $1^{\circ} 30^{\prime} 6^{\prime \prime}$ & 327 & $1^{\circ} 32^{\prime} 35^{\prime \prime}$ \\
\hline \multirow[t]{2}{*}{280} & $1^{\circ} 29^{\prime} 45^{\prime \prime}$ & 328 & $1^{\circ} 32^{\prime} 14^{\prime \prime}$ \\
\hline & & 329 & $1^{\circ} 31^{\prime} 49^{\prime \prime}$ \\
\hline 281 & $1^{\circ} 29^{\prime} 27^{\prime \prime}$ & 330 & $1^{\circ} 31^{\prime} 26^{\prime \prime}$ \\
\hline 282 & $1^{\circ} 29^{\prime} 19^{\prime \prime}$ & & \\
\hline 283 & $1^{\circ} 29^{\prime} 13^{\prime \prime}$ & 331 & $1^{\circ} 31^{\prime} 0^{\prime \prime}$ \\
\hline 284 & $1^{\circ} 29^{\prime} 18^{\prime \prime}$ & 332 & $1^{\circ} 30^{\prime} 38^{\prime \prime}$ \\
\hline 285 & $1^{\circ} 29^{\prime} 28^{\prime \prime}$ & 333 & $1^{\circ} 30^{\prime} 15^{\prime \prime}$ \\
\hline 286 & $1^{\circ} 29^{\prime} 48^{\prime \prime}$ & 334 & $1^{\circ} 29^{\prime} 55^{\prime \prime}$ \\
\hline 287 & $1^{\circ} 30^{\prime} 6^{\prime \prime}$ & 335 & $1^{\circ} 29^{\prime} 35^{\prime \prime}$ \\
\hline 288 & $1^{\circ} 30^{\prime} 37^{\prime \prime}$ & 336 & $1^{\circ} 29^{\prime} 19^{\prime \prime}$ \\
\hline 289 & $1^{\circ} 31^{\prime} 5^{\prime \prime}$ & 337 & $1^{\circ} 29^{\prime} 5^{\prime \prime}$ \\
\hline \multirow[t]{2}{*}{290} & $1^{\circ} 31^{\prime} 41^{\prime \prime}$ & 338 & $1^{\circ} 29^{\prime} \quad 1^{\prime \prime}$ \\
\hline & & 339 & $1^{\circ} 29^{\prime} 3^{\prime \prime}$ \\
\hline 291 & $1^{\circ} 32^{\prime} 9^{\prime \prime}$ & 340 & $1^{\circ} 29^{\prime} 17^{\prime \prime}$ \\
\hline 292 & $1^{\circ} 32^{\prime} 39^{\prime \prime}$ & & \\
\hline 293 & $1^{\circ} 32^{\prime} 57^{\prime \prime}$ & 341 & $1^{\circ} 29^{\prime} 43^{\prime \prime}$ \\
\hline 294 & $1^{\circ} 33^{\prime} 13^{\prime \prime}$ & 342 & $1^{\circ} 30^{\prime} 12^{\prime \prime}$ \\
\hline 295 & $1^{\circ} 33^{\prime} 17^{\prime \prime}$ & 343 & $1^{\circ} 30^{\prime} 48^{\prime \prime}$ \\
\hline
\end{tabular}


Table I (continued)

\begin{tabular}{llll}
\hline Day & Inclination & Day & Inclination \\
344 & $1^{\circ} 31^{\prime} 22^{\prime \prime}$ & 355 & $1^{\circ} 32^{\prime} 25^{\prime \prime}$ \\
345 & $1^{\circ} 31^{\prime} 58^{\prime \prime}$ & 356 & $1^{\circ} 31^{\prime} 57^{\prime \prime}$ \\
346 & $1^{\circ} 32^{\prime} 30^{\prime \prime}$ & 357 & $1^{\circ} 31^{\prime} 35^{\prime \prime}$ \\
347 & $1^{\circ} 32^{\prime} 59^{\prime \prime}$ & 358 & $1^{\circ} 31^{\prime} 6^{\prime \prime}$ \\
348 & $1^{\circ} 33^{\prime} 20^{\prime \prime}$ & 359 & $1^{\circ} 30^{\prime} 43^{\prime \prime}$ \\
349 & $1^{\circ} 33^{\prime} 35^{\prime \prime}$ & 360 & $1^{\circ} 30^{\prime} 17^{\prime \prime}$ \\
350 & $1^{\circ} 33^{\prime} 35^{\prime \prime}$ & & \\
351 & $1^{\circ} 33^{\prime} 33^{\prime \prime}$ & 361 & $1^{\circ} 29^{\prime} 57^{\prime \prime}$ \\
352 & $1^{\circ} 33^{\prime} 20^{\prime \prime}$ & 362 & $1^{\circ} 29^{\prime} 35^{\prime \prime}$ \\
353 & $1^{\circ} 33^{\prime} 6^{\prime \prime}$ & 363 & $1^{\circ} 29^{\prime} 22^{\prime \prime}$ \\
354 & $1^{\circ} 32^{\prime} 44^{\prime \prime}$ & 364 & $1^{\circ} 29^{\prime} 10^{\prime \prime}$ \\
& & 365 & $1^{\circ} 29^{\prime} 3^{\prime \prime}$
\end{tabular}

\section{References}

Cassini, G. D.: 1693, Traité de l'origine et du progress de l'Astronomie, Paris.

Habibullin, Sh. T.: 1968, Soviet Astron. 12, 526.

Koziel, K.: 1967, Icarus 7, 27.

Moutsoulas, M.: 1971, 'Librations of the Lunar Globe', in Physics and Astronomy of the Moon (2nd edition), Academic Press, New York. 